\title{
Growth Control and Disease Mechanisms in Computational Embryogeny
}

\author{
Or Yogev \\ California Institute of \\ Technology \\ 1200 East California Blvd. \\ Pasadena, CA 91125 U.S.A. \\ or@caltech.edu
}

\author{
Andrew A. Shapiro \\ Jet Propulsion Laboratory \\ California Institute of \\ Technology \\ 1200 East California Blvd. \\ Pasadena, CA 91125 U.S.A. \\ Andrew.A.Shapiro@jpl.nasa.gov
}

\author{
Erik K. Antonsson \\ California Institute of \\ Technology \\ 1200 East California Blvd. \\ Pasadena, CA 91125 U.S.A. \\ erik@design.caltech.edu
}

\begin{abstract}
This paper presents novel approach to applying growth control and diseases mechanisms in computational embryogeny. Our method, which mimics fundamental processes from biology, enables individuals to reach maturity in a controlled process through a stochastic environment. Three different mechanisms were implemented; disease mechanisms, gene suppression, and thermodynamic balancing. This approach was integrated as part of a structural evolutionary model. The model evolved continuum 3-D structures which support an external load. By using these mechanisms we were able to evolve individuals that reached a fixed size limit through the growth process. The growth process was an integral part of the complete development process. The size of the individuals was determined purely by the evolutionary process where different individuals matured to different sizes. Individuals which evolved with these characteristics have been found to be very robust for supporting a wide range of external loads.
\end{abstract}

\section{Categories and Subject Descriptors}

Categories and subject descriptors:I.2.11 Distributed Artificial Intelligence [Intelligent agents].

\section{General Terms}

Algorithms, Design

\section{Keywords}

Genetic Algorithm, Indirect Encoding, Stresses, Finite Element, Artificial Cell

\section{INTRODUCTION}

Natural evolution has produced systems of fantastic complexity, robustness and adaptability. Recent research has

Copyright is held by the author/owner(s).

GECCO'08, July 12-16, 2008, Atlanta, Georgia, USA.

ACM 978-1-60558-130-9/08/07. shown that it is the combination of both evolution and development processes that have produced these remarkable results [2, 3, 1]. Embryogeny is the process of growth by which a genotype develops into a phenotype, and is central to the emerging understanding of the relationship between evolution and development. In this case the developmental stage is artificially stopped. In this paper we will present a novel approach which mimics the fundamental processes in nature and helps an organism to reach a limited size even in a high volatility environment.

\section{ARTIFICIAL MODEL}

In the work reported here, an artificial embryogeny of structures has been created which is an extension of previous work done by the authors [?].

\subsection{Metabolism and Thermodynamics}

A thermodynamic energy consideration is present in the model which balances the maintaining of the organism mass with the creation of new mass [?]. The amount of energy $E_{c}$ that each cell may consume, in a given time step $\Delta t$, is proportional to its metabolic rate $B_{c}$. Part of this energy is used for maintaining the existing phenotype while the remaining energy may be used for creation of new mass, as shown in Equation 1,

$$
E_{c}=E_{0} B_{c} \Delta t
$$

The cell's metabolic rate is proportional to the volume of the phenotype $S$. Assuming that the volume of the cells has small variation in the phenotype, the metabolic rate can be determined using Kleinberg's law, given in Equation 2 where $N_{c}$ represents the number of cells.

$$
B_{c} \propto \frac{S^{3 / 4}}{N_{c}}
$$

Every gene regulation consumes energy. By specifying the amount of energy, required for cell division gene, and by establishing $E_{0}$, a thermodynamic size limit can be specified for the phenotypes, as shown in Equation 3. The specification of energy needs to be determined by the user based on his experience with the model. Our experience suggest that the model is not sensitive to these definitions, 


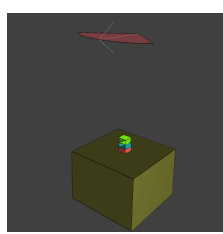

$\mathrm{t}=3$
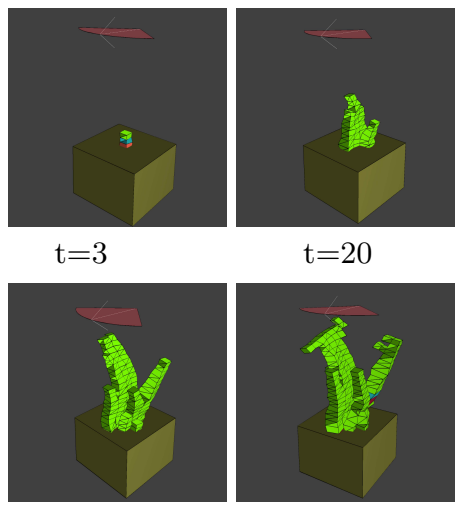

$\mathrm{t}=20$
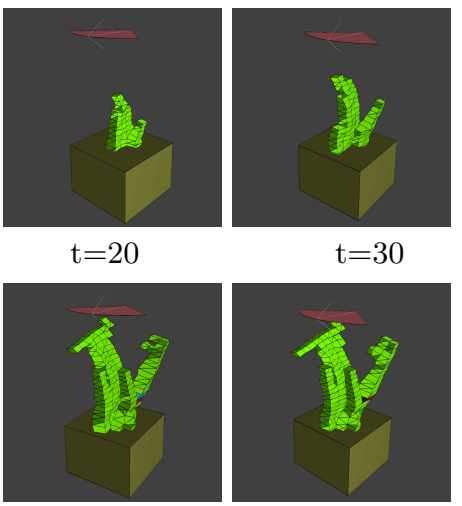

$\mathrm{t}=30$

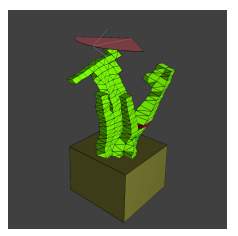

Figure 1: A growth process. The figure demonstrate a growth process of the best phenotype in the population in terms of performances. The colors in the images indicates the mechanical stress on the phenotype. Green indicates low stress while red indicate high stress. The creation of new mass was highly rated during the initial stages of development $(t=3$ $t=40$ ) and was decaying close to the maturity time. The phenotype was capable of supporting all three types of loads since no cell is over stressed.

$$
E_{c}=E_{0} \frac{S^{3 / 4}}{N_{c}}
$$

The advantage of using this approach is that there is no predefined upper bound or other limit on the size of the phenotype. Even when the phenotype reaches the thermodynamic limit, this approach will permit new mass to be created at the expense of removing existing mass, potentially changing the topology of the phenotype.

However, the thermodynamic balance will not prevent phenomena such as unlimited cell division or extermination of the entire phenotype. These last phenomena are addressed by evolution and disease mechanisms. Every phenotype may suffer from a disease during its developmental stage.

\section{EVOLUTIONARY SCHEME}

In Figure 2 the genome or the DNA of the phenotype is presented. The red and the yellow colors correspond to "veto" genes serving as control mechanism inside the genome. Although the genome itself is very complex, a large part of it is composed of control growth genes.

The evolutionary scheme is derived from a genetic algorithm. The algorithm is initialized with a set of randomly generated genomes. Starting from a single artificial cell, one individual is grown from each genome by executing the rules it contains.

\subsection{Structural Growth}

The approach outlined above has been applied an experiment representative of an important problem in engineering and nature. The problem was to synthesize the configuration of a structure to support a highly varied load generated by a wind. In addition, the structure needs to reach a certain height.

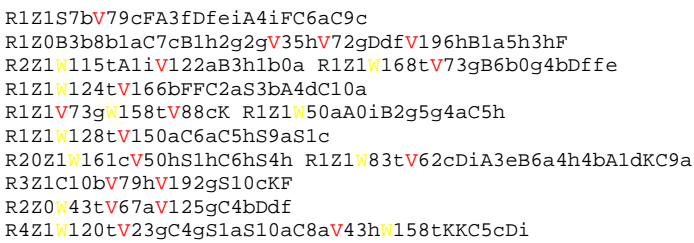

Figure 2: The genome of the phenotypes shown in Figure 1. The red and the yellow colors correspond to "veto" genes which control the development process of the phenotype.

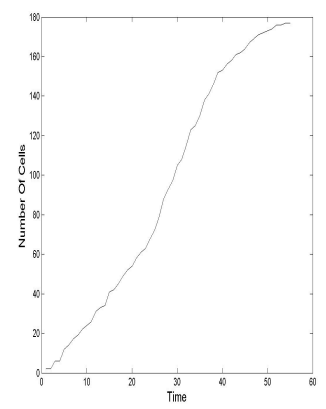

Figure 3: Growth plot - number of cells Vs. time.

The evaluation of the phenotypes was done only in their maturity stage. Figure 1 show the developmental process of phenotypes in Figure 1. which grew branches that tended to spread out. We can learn from figures 1 and 3 that new mass was created rapidly in early stages of development and decaying slowly as the phenotypes reached maturity.

\section{ACKNOWLEDGMENTS}

Our thanks to ACM SIGCHI for allowing us to modify templates they developed. The research described in this paper was sponsored by the Jet Propulsion Laboratory, California Institute of Technology, under a contract with the National Aeronautics and Space Administration as part of the Ultra-Reliability Program

\section{REFERENCES}

[1] P. J. Bentley and S. Kumar. Three ways to grow designs: A comparison of embryogenies for an evolutionary design problem. In W. Banzhaf, J. Daida, A. E. Eiben, M. H. Garzon, V. Honavar, M. Jakiela, and R. E. Smith, editors, Proceedings of the Genetic and Evolutionary Computation Conference, pages 35-43, Orlando, FL, 1999. Morgan Kaufmann.

[2] S. B. Carroll. Endless Forms Most Beautiful: The new science of evo devo and the making of the animal kingdom. W. W. Norton \& Co., 2005.

[3] A. R. Palmer. Symmetry breaking and the evolution of development. Science, 306(5697):828-833, Oct. 2004. 